\title{
A Multivariate Asymmetric Long Memory Conditional Volatility Model with X, Regularity and Asymptotics ${ }^{* *}$ \\ Manabu Asai ${ }^{1, ~}$ and Michael McAleer ${ }^{2 *}$ \\ ${ }^{1}$ Faculty of Economics \\ Soka University, Japan \\ ${ }^{2}$ Department of Quantitative Finance \\ National Tsing Hua University, Taiwan \\ *michael.mcaleer@gmail.com
}

\begin{abstract}
The paper derives a Multivariate Asymmetric Long Memory conditional volatility model with Exogenous Variables $(X)$, or the MALMX model, with dynamic conditional correlations, appropriate regularity conditions, and associated asymptotic theory. This enables checking of internal consistency and allows valid statistical inferences to be drawn based on empirical estimation. The underlying vector random coefficient autoregressive process, which has well established regularity conditions and associated asymptotic properties, is discussed, and a simple explanation is given as to why only the diagonal BEKK model, and not the Hadamard, triangular or full BEKK models, has regularity conditions and asymptotic properties. Various special cases, including the diagonal BEKK model of Baba et al. (1985) and Engle and Kroner (1995), VARMA-GARCH model of Ling and McAleer (2003), and VARMA-AGARCH model of McAleer et al. (2009), are discussed. There does not seem to have been a derivation of a univariate conditional volatility model with exogenous variables $(X)$ that has dynamic conditional correlations, appropriate regularity conditions, and associated asymptotic theory. Therefore, the derivation of a multivariate conditional volatility model with exogenous variables $(X)$ that has regularity conditions and asymptotic theory would seem to be a significant extension of the existing literature.
\end{abstract}

Keywords: Multivariate conditional volatility, Vector random coefficient autoregressive process, Asymmetry, Long memory, Exogenous variables, Dynamic conditional correlations, Regularity conditions, Asymptotic properties.

JEL: C22, C52, C58, G32.

\section{Introduction}

Asymmetry, long memory and exogenous variables (hereafter labelled as X) have been incorporated into financial returns, while both asymmetry and long memory have been used widely in financial volatility models.

Asymmetry can be incorporated into dynamic financial returns and associated volatility models to show that positive and negative returns on financial assets typically have different volatility, with negative returns tending to have greater volatility than positive returns of similar magnitude. Asymmetry has been considered in several conditional, stochastic, and realized volatility models.

\footnotetext{
** The authors are grateful to Chia-Ling Chang for helpful discussions. The first author acknowledges the financial support of the Japan Ministry of Education, Culture, Sports, Science and Technology, Japan Society for the Promotion of Science, and Australian Academy of Science. The second author is most grateful for the financial support of the Australian Research Council, National Science Council, Ministry of Science and Technology (MOST), Taiwan, Japan Society for the Promotion of Science, and Institute of Advanced Sciences, Yokohama National University. McAleer is University Distinguished Research Professor at the Institute for Social and Economic Sciences (ISES), Dhurakij Pundit University (DPU), Bangkok, Thailand.
} 
Numerous instances of asymmetry have been considered for multivariate stochastic volatility models (see, for example, Asai et al. (2006), Asai and McAleer (2006, 2009a, 2009b, 2011)), but not as frequently for conditional volatility (see McAleer et al., 2009) and realized volatility models (see, for example, Asai and McAleer (2015), Asai et al. (2012), Asai et al. (2011a, 20011b)).

Long memory models are useful when the autocorrelation function (ACF) of returns converges slowly to zero. If a stochastic process has a unit variance, the use of first differences may be convenient. When the ACF exhibits slowly declining correlations that do not converge to zero, a fractional differencing or long memory model may be useful (see, for example, Asai and McAleer (2015)). This will be discussed further in Section 3.

Exogenous variables have been used widely in both univariate and multivariate conditional volatility models, but the regularity conditions and asymptotic theory associated with QuasiMaximum Likelihood Estimation (QMLE) do not seem to have been analysed. This will also be discussed further in Section 3.

A primary purpose of the paper is to incorporate asymmetry, long memory and exogenous variables (X) into multivariate conditional volatility models in a dynamic model of conditional covariances, and to provide the regularity conditions and asymptotic theory associated with the Quasi-Maximum Likelihood Estimation (QMLE).

The plan of the remainder of the paper is as follows. Section 2 discusses the regularity conditions and asymptotic theory of vector random coefficient autoregressive processes. A multivariate asymmetric long memory conditional volatility model with exogenous variables, X (MALMX), regularity conditions and asymptotic theory is developed in Section 3. The novelty and various special cases of MALMX are analysed. Some concluding remarks are presented in Section 4.

\section{Regularity Conditions and Asymptotic Theory of Vector Random Coefficient Autoregressive Processes}

Consider the conditional mean of financial returns as follows:

$$
y_{t}=E\left(y_{t} \mid I_{t-1}\right)+\varepsilon_{t}
$$

where the $m \times 1$ vector of returns, $y_{t}=\Delta \log P_{t}$, represents the log-difference in financial asset prices $\left(P_{t}\right), I_{t-1}$ is the information set at time $t-1$, and $\varepsilon_{t}$ is an $m \times 1$ vector of conditionally heteroskedastic shocks. If the conditional mean in equation (1) is a vector ARMA process, the conditions given in Ling and McAleer (2003) would need to be examined for restrictions on the parameters and variables.

Consider the simple vector random coefficient autoregressive process given by:

$$
\varepsilon_{t}=\theta_{t} \varepsilon_{t-1}+\eta_{t}
$$

in which there is no asymmetry, long memory or exogenous variables. In equation (2), the regularity conditions are given for the case where:

$$
\begin{aligned}
& \varepsilon_{t} \text { and } \eta_{t} \text { are } m \times 1 \text { vectors, } \\
& \theta_{t} \text { is an } m \times m \text { matrix, } \\
& \theta_{t} \sim \operatorname{iid}(0, A),
\end{aligned}
$$

$A$ is a diagonal $m \times m$ matrix for conformability of matrix multiplication in the derivation of the conditional covariance matrix of $\varepsilon_{t}$. 
Lack of diagonality of the covariance matrix $A$ means that only a diagonal multivariate conditional volatility model, and not the Hadamard, triangular or full counterparts, has regularity conditions that are consistent with the vector random coefficient autoregressive process given in equation (2). Consequently, only a diagonal multivariate conditional volatility model can have asymptotic properties.

For purposes of Quasi-Maximum Likelihood Estimation (QMLE), in which the errors are not normal, as assumed, invertibility of the function can be shown by rewriting equation (1) as:

$\eta_{t}=\varepsilon_{t_{-}} \theta_{t} \varepsilon_{t-1}$

in which $\eta_{t}$ is a function of the data as it is a function of $\varepsilon_{t}$, which is a function of the data, and the values of $\theta_{t}$ can be determined through simulation.

In general, the proofs of the asymptotic properties follow from the fact that the alternative multivariate conditional volatility models can be derived from a vector random coefficient autoregressive process. For a general analysis of the estimation of multivariate random coefficient autoregressive models, see Nicholls and Quinn (1981). McAleer et al. (2008) provide a general proof of multivariate models that are based on proving that the regularity conditions satisfy the conditions given in Jeantheau (1998) for consistency, and the conditions given in Theorem 4.1.3 in Amemiya (1985) for asymptotic normality.

\section{Multivariate Asymmetric Long Memory Conditional Volatility Model with X (MALMX), Regularity Conditions and Asymptotic Theory}

In this section, we derive a new Multivariate Asymmetric Long Memory conditional volatility model with exogenous regressors $(X)$, or the MALMX model, with dynamic conditional correlations, and consider various special cases, including the diagonal BEKK model of Baba et al. (1985) and Engle and Kroner (1995), VARMA-GARCH model of Ling and McAleer (2003), and VARMA-AGARCH model of McAleer et al. (2009).

A simple fractionally integrated model that incorporates fractional differencing into an $\operatorname{ARMA}(p, q)$ process is given as an ARFIMA $(p, d, q)$, in which the fractionally integrated component has difference parameter, $d$, where $d$ is a value such that $|d|<0.5$. We will refer to such a model with $F I\left({ }^{\varepsilon_{t-1}}\right)$, where $\varepsilon_{t-1}$ is the lagged return shock, as a fractionally integrated long memory process. Unlike the orders of the autoregressive and moving average components, $p$ and $q$, respectively, the differencing parameter, $d$, can take non-integer values.

Following from the conditional mean of financial returns given in equation (1), in order to derive conditional volatility specifications, it is necessary to specify the stochastic processes underlying the returns shocks, ${ }^{\varepsilon_{t}}$. Suppose that the $m \times 1$ vector of returns shocks is given by the following vector random coefficient autoregressive process:

$$
\varepsilon_{t}=\theta_{t} \varepsilon_{t-1}+\phi_{t} \varepsilon_{t-1} I\left({ }^{\varepsilon_{t-1}}\right)+{ }^{t} F I\left({ }^{t-1}\right)+\xi_{t} \sqrt{X_{t-1}}+\eta_{t}
$$

where 


$$
\begin{aligned}
& \varepsilon_{t}, \eta_{t} \text { and } X_{t} \text { are } m \times 1 \text { vectors, } \\
& \theta_{t}, \phi_{t}, \psi_{t}, \text { and } \xi_{t} \text { are } m^{\times} m \text { matrices, } \\
& \theta_{t} \sim \operatorname{iid}(0, A), \\
& \phi_{t} \sim \operatorname{iid}(0, B), \\
& \psi_{t} \sim \operatorname{iid}(0, C), \\
& \xi_{t} \sim \operatorname{iid}(0, D), \\
& \eta_{t} \sim \operatorname{iid}(0, W),
\end{aligned}
$$

$A, B, C$ and $D$ are diagonal $m \times m$ matrices for conformability of matrix multiplication in the derivation of the conditional covariance matrix of $\varepsilon_{t}$,

$F I\left({ }^{\varepsilon_{t-1}}\right)$ is a fractionally integrated long memory process,

$I\left({ }^{\varepsilon_{t-1}}\right)$ is an indicator variable that has the value of unity for a negative shock in an element of $\varepsilon_{t-1}$, and zero for a positive shock,

$X_{t-1}$ is a positive-valued exogenous variable,

$\sqrt{X_{t-1}}$ denotes the square root of each element of $X_{t-1}$.

As $\theta_{t}, \phi_{t}, \psi_{t}$, and $\xi_{t}$ are $m \times m$ matrices, their respective covariance matrices can have dimension as large as $m^{2} \times m^{2}$, or as small as $\frac{1}{2} m(m+1) \times \frac{1}{2} m(m+1)$ if the respective covariance matrices are symmetric.

In what follows, for conformability of matrix multiplication in the derivation of the conditional covariance matrix of $\varepsilon_{t}$, it is necessary that $A, B, C$ and $D$ are diagonal $m \times m$ matrices. This means that the resulting multivariate conditional volatility models will be restricted in terms of parameterizations.

It follows from equation (4) that:

$$
\begin{aligned}
& H_{t} \equiv E\left(\left.\varepsilon_{t} \varepsilon_{t^{\prime}}\right|^{I t-1}\right) \\
& =W+A^{\varepsilon_{t-1}} \varepsilon_{t-1} A^{\prime}+B^{\varepsilon_{t-1}} \varepsilon_{t-1}^{\prime} B^{\prime}\left[I\left({ }^{\varepsilon_{t-1}}\right)\right]+C F I\left({ }^{\varepsilon_{t-1}}\right) F I\left({ }^{\varepsilon_{t-1}}\right)^{\prime} C^{\prime}+D^{X_{t-1}} X_{t-1} D^{\prime}
\end{aligned}
$$

where $I_{t-1}$ is the information set available at time $t$-1. Equation (5) is a new multivariate asymmetric long memory conditional volatility model with exogenous variables, $X$, hereafter the MALMX model. 
The new model has appropriate regularity conditions and asymptotic theory, as it can be derived from a vector random coefficient autoregressive process. The regularity conditions satisfy the conditions given in Jeantheau (1998) for consistency, and the conditions given in Theorem 4.1.3 in Amemiya (1985) for asymptotic normality.

It is useful to define the multivariate extension of the relationship between the returns shocks and the standardized residuals, that is, $\eta_{t}=\varepsilon_{t} / \sqrt{h_{t}}$ in the univariate context, that is, where $\eta_{t}, \varepsilon_{t}$, and $h_{t}$ are scalars. The multivariate definition of the relationship between $\varepsilon_{t}$ and $\eta_{t}$ is given as:

$$
\varepsilon_{t}=D_{t}^{1 / 2} \eta_{t}
$$

where $D_{t}=\operatorname{diag}\left(h_{1 t}, h_{2 t}, \ldots, h_{m t}\right)$ is a diagonal matrix comprising the univariate conditional volatilities, so that $D_{t}^{1 / 2}$ has conditional standard deviations as typical elements along the main diagonal. Define the conditional covariance matrix of $\varepsilon_{t}$ as $H_{t}$, as in equation (5). As the $m \times 1$ vector, $\eta_{t}$, is assumed to be iid for all $m$ elements, the conditional correlation matrix of $\eta_{t}$, which is equivalent to the conditional correlation matrix of $\eta_{t}$, is given by $\Gamma_{t}$. Therefore, the conditional expectation of (6) is defined as:

$$
H_{t}=D_{t}^{1 / 2} \Gamma_{t} D_{t}^{1 / 2}
$$

Equivalently, the conditional correlation matrix, ${ }^{\Gamma_{t}}$, can be defined as:

$$
\Gamma_{t}=D_{t}^{-1 / 2} H_{t} D_{t}^{-1 / 2} \text {. }
$$

Equation (7) is useful if a model of $\Gamma_{t}$ is available for purposes of estimating ${ }^{H_{t}}$, whereas equation (8) is useful if a model of $H_{t}$ is available for purposes of estimating $\Gamma_{t}$.

\subsection{Special Cases}

(i) When $B=C=D=0$, the null matrix (see McAleer et al. (2008) for a derivation), this gives the diagonal BEKK model, which is also an extension of the VARMA-GARCH model of Ling and McAleer (2003) with static conditional correlations, that is, where $\Gamma_{t}$ in equations (7) and (8) is a constant matrix $\Gamma$.

(ii) As the matrices of coefficients in equation (5), namely $A, B, C$ and $D$, are all diagonal, this means that only the diagonal BEKK model, and not the Hadamard, triangular or full BEKK models, has appropriate regularity conditions and associated asymptotic theory.

(iii) When $C=D=0$, the null matrix, this gives a multivariate asymmetric dynamic conditional covariance model, which is an extension of the symmetric diagonal BEKK model, an extension of the asymmetric VARMA-AGARCH model of McAleer et al. (2009) in which the conditional correlations are static, and an extension of the symmetric VARMA-GARCH model of McAleer et al. (2008) in which the conditional correlations are static. 
(iv)The case $C=D=0$ with dynamic conditional covariances is an extension of the VARMA-AGARCH model of McAleer et al. (2009), in which the conditional correlations are static.

(v) When $C \neq 0$ and $D \neq 0$, the derivation of the MALMX model is a new multivariate conditional volatility model with long memory and exogenous variables $(X)$, with dynamic conditional correlations, appropriate regularity conditions, and associated asymptotic theory.

\subsection{Novelty of MALMX}

There does not yet seem to have been a derivation of a univariate conditional volatility model with exogenous variables $(X)$ that has appropriate regularity conditions and associated asymptotic theory. Therefore, the derivation of a multivariate conditional volatility model with exogenous variables $(X)$ that has dynamic conditional correlations, appropriate regularity conditions and associated asymptotic theory, would seem to be a significant extension of the existing literature on conditional volatility models.

\section{Concluding Remarks}

The paper derived a Multivariate Asymmetric Long Memory conditional volatility model with Exogenous Variables $(X)$, or the MALMX model, with dynamic conditional correlations, appropriate regularity conditions and associated asymptotic theory. This enables checking of internal consistency and allows valid statistical inferences to be drawn based on empirical estimation.

The underlying vector random coefficient autoregressive process, which has well established regularity conditions and asymptotic properties, was discussed, and a simple explanation was given as to why only the diagonal BEKK model, and not the Hadamard, triangular or full BEKK models, has appropriate regularity conditions and associated asymptotic theory.

Various special cases, including the diagonal BEKK model of Baba et al. (1985) and Engle and Kroner (1995), which has dynamic conditional correlations, VARMA-GARCH model of Ling and McAleer (2003), and VARMA-AGARCH model of McAleer et al. (2009), both of which have static conditional correlations, were discussed.

The new MALMX model should be useful addition to the only existing multivariate conditional volatility model with dynamic conditional correlations, appropriate regularity conditions, and associated asymptotic theory, namely the diagonal BEKK model.

\section{References}

Amemiya, T. (1985), Advanced Econometrics, Harvard University Press, Cambridge, MA, USA.

Asai, M. and M. McAleer (2006), Asymmetric multivariate stochastic volatility, Econometric Reviews, 25, 453-473.

Asai, M. and M. McAleer (2009a), The structure of dynamic correlations in multivariate stochastic volatility models, Journal of Econometrics, 150, 182-192.

Asai, M. and M. McAleer (2009b), Multivariate stochastic volatility, leverage and news impact surfaces, Econometrics Journal, 12, 292-309. 
Asai, M. and M. McAleer (2011), Alternative asymmetric stochastic volatility models, Econometric Reviews, 30, 548-564.

Asai, M. and M. McAleer (2015), Forecasting co-volatilities via factor models with asymmetry and long memory in realized covariance, Journal of Econometrics, 189, 251-262.

Asai, M., M. McAleer and M. Medeiros (2012a), Estimation and forecasting with noisy realized volatility, Computational Statistics \& Data Analysis, 56, 217-230.

Asai, M., M. McAleer and M. Medeiros (2012b), Asymmetry and long memory in volatility modeling, Journal of Financial Econometrics, 10, 495-512.

Asai, M., M. McAleer, and J. Yu (2006), Multivariate stochastic volatility: A review, Econometric Reviews, 25, 145-175.

Baba, Y., R.F. Engle, D. Kraft and K.F. Kroner (1985), Multivariate simultaneous generalized ARCH. Unpublished manuscript, Department of Economics, University of California, San Diego, CA, USA.

Engle, R.F. and K.F. Kroner (1995), Multivariate simultaneous generalized ARCH, Econometric Theory, 11, 122-150.

Glosten, L.R., R. Jagannathan and D.E. Runkle (1992), On the relation between the expected value and volatility of nominal excess return on stocks, Journal of Finance, 46, 1779-1801.

Jeantheau, T. (1998), Strong consistency of estimators for multivariate ARCH models, Econometric Theory, 14, 70-86.

Ling, S. and M. McAleer (2003), Asymptotic theory for a vector ARMA-GARCH model, Econometric Theory, 19, 278-308.

McAleer, M., F. Chan, S. Hoti and O. Lieberman (2008), Generalized autoregressive conditional correlation, Econometric Theory, 24(6), 1554-1583.

McAleer, M., S. Hoti and F. Chan (2009), Structure and asymptotic theory for multivariate asymmetric conditional volatility, Econometric Reviews, 28, 422-440.

Nicholls, D.F. and B.G. Quinn (1981), The estimation of multivariate random coefficient autoregressive models, Journal of Multivariate Analysis, 11(4), 544-555. 